Projets

de paysage

\section{Projets de paysage}

Revue scientifique sur la conception et l'aménagement de l'espace

\section{$11 \mid 2014$}

Paysage(s) et patrimoine(s)

\title{
Le Paysage, outil de résistance face à l'urbanisation
}

Quelques enseignements dans les vignobles de Vouvray et de Montlouis sur Loire

The Landscape, a tool for resisting urbanisation - Lessons learned in the vineyards of Vouvray and Montlouis sur Loire

Jean-Louis Yengué et Clothilde Chaballier

\section{(2) OpenEdition}

\section{Journals}

Édition électronique

URL : http://journals.openedition.org/paysage/11208

ISSN : 1969-6124

\section{Éditeur :}

École nationale supérieure du paysage de Versailles-Marseille, Institut national des sciences appliquées Centre Val de Loire - École de la nature et du paysage, École nationale supérieure d'architecture et de paysage de Bordeaux, École nationale supérieure d'architecture et de paysage de Lille, Agrocampus Angers

Référence électronique

Jean-Louis Yengué et Clothilde Chaballier, "Le Paysage, outil de résistance face à l'urbanisation », Projets de paysage [En ligne], 11 | 2014, mis en ligne le 31 décembre 2014, consulté le 29 septembre 2020. URL : http://journals.openedition.org/paysage/11208 


\section{Le Paysage, outil de résistance face à l'urbanisation}

Quelques enseignements dans les vignobles de Vouvray et de Montlouis sur Loire

The Landscape, a tool for resisting urbanisation - Lessons learned in the

vineyards of Vouvray and Montlouis sur Loire

Jean-Louis Yengué et Clothilde Chaballier

\section{Introduction : contexte et questionnement}

1 Aujourd'hui, le milieu urbain regroupe déjà plus de la moitié de la population mondiale, et des prévisions démographiques prévoient, pour 2020, que $80 \%$ des Européens vivront dans des espaces urbains (Laroche et al., 2006, Cavailhes, 2004). En France ce sont environ 60000 hectares, soit l'équivalent d'un département français tous les 10 ans, qui sont artificialisés et cela principalement au détriment des zones agricoles et naturelles et le phénomène est en voie d'accélération. Le problème se pose avec acuité sur le territoire du schéma de cohérence territoriale de Tours (Scot approuvé en septembre 2013) que nous qualifierons d'agglomération tourangelle. Il regroupe 40 communes sur $830 \mathrm{~km}^{2}$ (13,5\% de la superficie de l'Indre-et-Loire) pour 353000 habitants (61\% de la population départementale). L'agglomération n'a cessé de s'accroître depuis la fin de la Seconde Guerre mondiale avec une accélération à partir des années 1970. Le diagnostic du Scot fait état, depuis 30 ans, d'une perte de 8000 ha de surface agricole utile et de $58 \%$ des exploitations agricoles. Malgré tout, le secteur agricole tente de lutter contre l'étalement urbain. C'est le cas du vignoble qui ne représente plus qu'à peine $5 \%$ du territoire. Les nombreuses vignes qui existaient ici avant la Seconde Guerre mondiale, étudiées par Babonaux (1967) et Dion (1934), ont été très fortement réduites (Yengué et al., 2003). Seuls ne résistent que les vignobles de Vouvray et de Montlouis-sur-Loire. Ces dernières sont deux communes périurbaines à 
une quinzaine de kilomètres de Tours, qui, malgré la présence de leurs vignobles, subissent une très forte pression foncière liée à l'urbanisation.

2 Si de nombreux travaux ont montré que la viticulture résiste mieux à l'urbanisation que les autres formes d'agriculture, car elle produit des revenus hautement plus importants qu'une agriculture classique (Barthélémy et al., 2000, Pères, 2007, 2009, Chaballier, 2010), nous souhaitons, à travers le cas de ces deux vignobles, comprendre la place du paysage dans cette résistance.

\section{Approche méthodologique}

Les vignobles AOC de Montlouis et de Vouvray sont situés dans le Val de Loire, à l'Ouest de Tours.

Figure 1. Les AOC Vouvray et Montlouis-sur-Loire dans leur contexte territorial

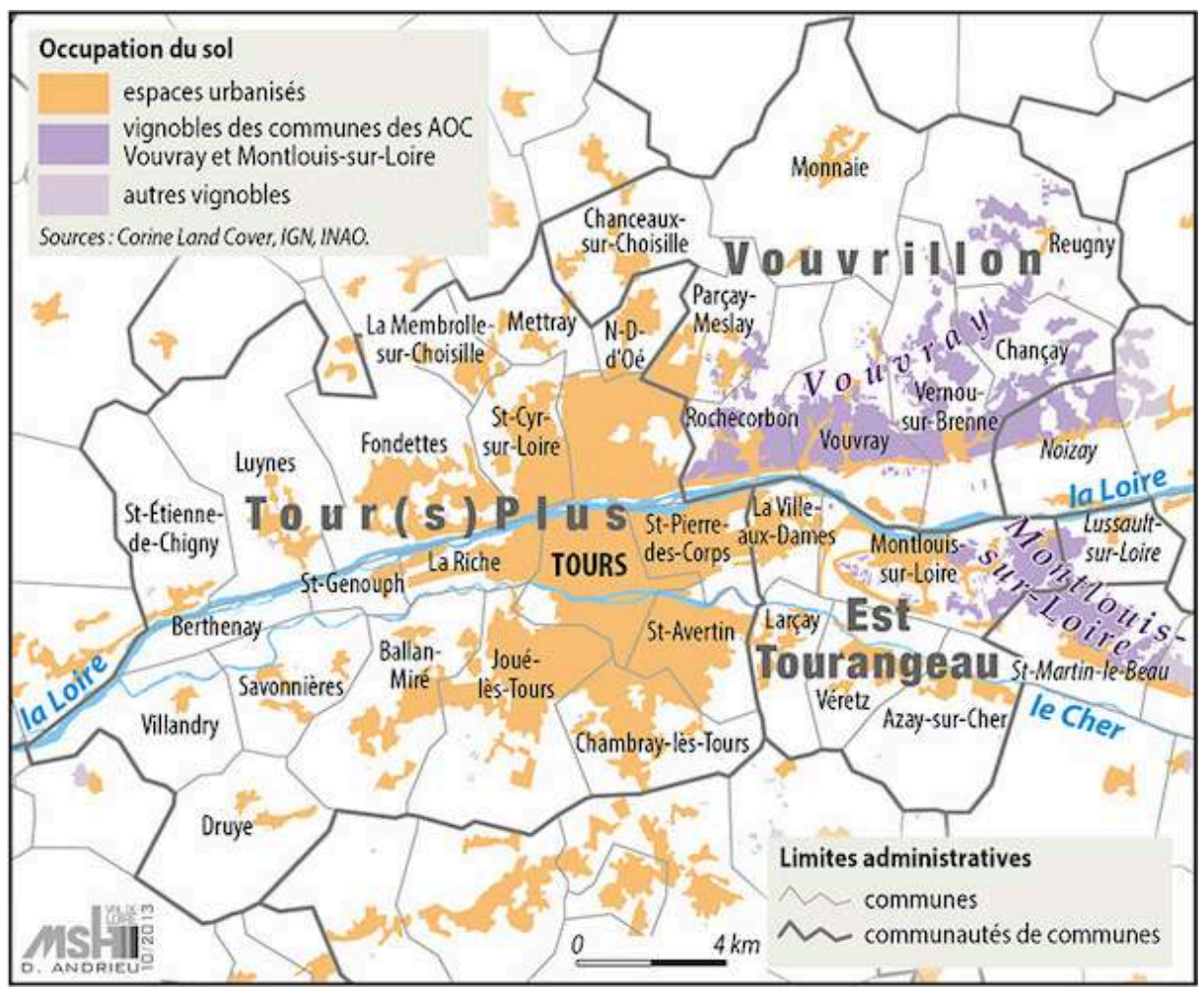

4 L'appellation Vouvray date de 1936. Elle couvre aujourd'hui environ 2000 ha sur le coteau nord de la Loire, répartis sur 8 communes (Chançay, Noizay, Parçay-Meslay, Sainte-Radegonde-en-Touraine - rattachée à Tours en 1964 -, Reugny, Rochecorbon, Vernou-sur-Brenne et Vouvray) et concerne un peu moins de 200 viticulteurs. La production annuelle est d'environ $115000 \mathrm{hl}$ composée de $40 \%$ en vins tranquilles et $60 \%$ en vins fines bulles ${ }^{1}$.

5 L'AOC Montlouis, quant à elle, est née par décret, le 6 décembre 1938. Jusqu'alors, elle partageait avec l'AOC Vouvray la dénomination de Vins de Vouvray. Le décret du 19 novembre 2002 remplace le nom de Montlouis par Montlouis-sur-Loire. L'appellation, située entre la Loire et le Cher, s'étend sur les communes de Montlouissur-Loire, Saint-Martin-le-Beau et Lussault-sur-Loire et couvre environ 385 ha pour 72 
viticulteurs ${ }^{2}$. La production annuelle oscille autour de $17000 \mathrm{hl}$ dont $55 \%$ de vins tranquilles et $45 \%$ de fines bulles, comme à Vouvray

6 Ces deux AOC, malgré leur taille bien différente, se trouvent dans le même contexte territorial. Elles font partie du Scot de Tours et sont incluses dans des établissements publics de coopération intercommunale (la communauté de communes du Vouvrillon [CCV] pour AOC Vouvray et la communauté de communes de l'Est tourangeau [CCET] pour une grande partie du vignoble de Montlouis-sur-Loire) qui souhaitent garder leur autonomie face à Tour(s)Plus, la communauté d'agglomération de Tours

7 Ces vignobles et leur paysage sont au cœur d'enjeux que nous avons étudiés par une série d'entretiens semi-directifs. Dans une démarche plus qualitative que quantitative, nous avons interrogé trois catégories d'acteurs. Ces entretiens ont pris la forme de longs échanges d'environ deux heures, guidés par des questions au préalablement identifiées et différentes d'un acteur à l'autre :

- Les acteurs publics, c'est-à-dire les communes (maires et/ou adjoints) des appellations ainsi que la CCET et la CCV afin de connaître les politiques, les plans d'action en vigueur et les éventuels projets concernant la place de l'agriculture, et plus particulièrement du vignoble, au sein de leur territoire. Nous avons cherché à savoir quelles étaient leurs actions pour protéger le vignoble, s'il y en avait, et pourquoi elles avaient été mises en place; de quelle façon et dans quel but, ainsi que les résultats attendus et/ou obtenus. Pour finir, nous les avons interrogés sur leurs perspectives d'avenir.

- La profession des vignerons à travers les syndicats de vignerons des deux AOC. Les présidents ont été interrogés afin de connaître les actions mises en place pour défendre la profession et protéger l'AOC. Il s'agissait aussi d'avoir leurs points de vue sur les décisions politiques en matière d'urbanisation et/ou de protection du vignoble, et de connaître leurs craintes et leurs souhaits dans ce contexte de montée de l'urbanisation. L'objectif était aussi de percevoir les points forts et les limites des moyens de résistance mis en place par les collectivités, mais aussi de se rendre compte de la convergence (ou pas) des intérêts de chacun.

- Enfin, des viticulteurs ont été interrogés. Nous en avons aléatoirement sélectionné cinq dans chaque $\mathrm{AOC}^{3}$. Nous avons cherché à savoir quelles étaient leurs stratégies pour défendre leurs propres intérêts. Se sentaient-ils concernés par la protection de la viticulture et comment appréciaient-ils les actions mises en œuvre tant par les collectivités que par les syndicats?

\section{Des stratégies de résistance divergentes}

8 L'analyse des discours suite aux entretiens, mais aussi l'exploitation de divers documents (presse locale, bulletins municipaux, etc.) font clairement ressortir deux stratégies bien différentes entre les deux AOC.

\section{L'AOC Montlouis : une action coordonnée entre les acteurs}

9 Des trois communes de l'AOC Montlouis-sur-Loire, seule la municipalité de Montlouis s'investit fortement pour la préservation de son vignoble. Les deux autres (SaintMartin-le-Beau et Lussault-sur-Loire), plus rurales, sont davantage en retrait. Les questions d'artificialisation des sols se posent chez elles avec moins d'acuité. 
10 Dès la fin des années 1970, la municipalité de Montlouis-sur-Loire a œuvré pour le maintien de la viticulture en milieu périurbain. Les plans d'occupation des sols (POS) de 1983, 1995 et 1999 ont protégé le vignoble en classant une partie en zone strictement inconstructible, volonté qui est confirmée dans le PLU (approuvé en 2012).

11 Mais, selon la municipalité, la protection apportée par ces documents d'urbanisme n'est pas suffisante face à la forte pression foncière, notamment au bord des hameaux et sur les franges urbaines. En effet, le PLU, et le classement en zone A, n'est pas jugé suffisant car il est soumis à des révisions, procédures qui peuvent être activées à l'initiative des communes seules.

12 De plus, depuis quelques années, l'envolée des prix du foncier constructible a accéléré une nouvelle spéculation qui impacte les exploitants non propriétaires et ceux qui souhaitent s'installer. Ils ne peuvent plus ni louer ni acheter dans les secteurs proches (voire plus lointains) des zones urbanisées.

13 Alors, les élus de la ville ont souhaité renforcer la protection en proposant une zone agricole protégée (ZAP). L'outil zone agricole protégée est défini dans le code rural à l'article L.112-2 comme « des zones dont la préservation présente un intérêt général en raison soit de la qualité de leur production, soit de leur situation géographique pouvant faire l'objet d'un classement en tant que zones agricoles protégées ». L'objet d'une ZAP est d'ériger la "vocation agricole » d'une telle zone en "servitude d'utilité publique » et donc de la soustraire aux aléas des fluctuations du droit des sols, inhérentes au mode même de production des Scot et des PLU.

Ce projet de ZAP a été une initiative de la commune (maître d'ouvrage) et de la CCET (assistant maître d'ouvrage). La CCET s'est fait accompagner d'un comité technique composé de la ville de Montlouis-sur-Loire, de l'agence d'urbanisme et de l'agglomération de Tours, du syndicat des vins et de l'Institut national des appellations d'origine. Cette ZAP vise à protéger le vignoble et à rassurer les vignerons :

"C'est un engagement avec eux. En effet, la ville souhaitait urbaniser un secteur viticole de 35 hectares et en échange on a créé 6 zones agricoles protégées. De plus, de nombreux terrains étaient laissés en friche en attendant d'être constructibles (parcelles viticoles ou autres) et la mise en place de la ZAP avait aussi pour but d'empêcher ce phénomène. » (Mairie de Montlouis-sur-Loire.)

Le périmètre de la zone agricole protégée résulte d'un compromis entre le syndicat viticole et la municipalité (Serrano, Vianey, 2007). Le syndicat avait une position maximaliste. Il demandait que l'intégralité de l'aire d'appellation d'origine soit protégée.

«Le président du syndicat voulait que toute l'AOC soit classée en ZAP. Mais ce n'est pas possible, il faut être conscient que l'on n'empêchera pas la commune de s'étendre, même si ce n'est pas sur la ZAP. Mais il faut mesurer aussi que c'est une ville qui est à la périphérie de Tours et qui va s'étendre. Je n'étais pas d'accord avec la position du syndicat des vins par rapport à des délimitations un peu trop draconiennes, parce que je préférais qu'on abandonne des parties qui étaient appelées à voir de moins en moins de vignes. Je pense notamment au sud de la commune, où j'ai connu étant petit des grandes zones de vigne, et aujourd'hui il y en a pratiquement plus. » (Monsieur M., viticulteur à Montlouis-sur-Loire.)

La municipalité avait quant à elle une vision très réduite du périmètre de protection. Elle souhaitait le limiter aux secteurs proches du centre urbain de Montlouis, soumis à la plus forte pression urbaine. Sous la pression du syndicat viticole, elle a accepté de protéger une grande partie de l'AOC. 

communal, soit 322,5 hectares et intègre la quasi-totalité (environ $80 \%$ ) des limites de l'appellation Montlouis-sur-Loire. Ces secteurs ont été sélectionnés suivant trois critères : être classé en AOC Montlouis (l'AOC Touraine n'est pas intégrée au périmètre), présenter un enjeu paysager riche et être dans des espaces où la pression foncière est forte. Le projet a été validé en conseil municipal et présenté à la population en 2006. Le 17 août 2007, la zone agricole protégée est officiellement créée par arrêté préfectoral.

Même si elle n'a pas été à l'initiative de ce projet, le rôle de la profession a été très important dans cette démarche :

«Le but c'était de pouvoir avoir un bon diagnostic et vraiment les intentions de chacun. Il y a eu un recensement auprès de la profession [...]. Sinon, par la suite, elle a participé à la définition du périmètre. L'Institut national de l'origine et de la qualité (Inao) a aussi joué un rôle important, parce qu'il y a des zones AOC qui n'étaient pas dans le périmètre, et ce sont eux qui, sur certaines parcelles, ont agrandi le périmètre. » (CCET.)

Les agriculteurs n'ont donc pas émis de demandes particulières et ont accepté la mise en place de la ZAP. Les seules oppositions contre ce projet ont été consécutives à des problèmes de succession : des agriculteurs qui partaient à la retraite souhaitaient faire une plus-value sur leurs terres. Pour les vignerons, la ZAP n'apporte aucune contrainte (sauf pour ceux qui souhaitent vendre leurs terres). Elle ne change pas les habitudes des vignerons.

«On ne peut pas dire que ça apporte des contraintes. Non, ça n'apporte aucune contrainte, simplement respecter les délimitations. Je pense que ça apporte plus de contraintes à la municipalité. » (Monsieur M., viticulteur à Montlouis-sur-Loire.)

Économiquement elle ne change rien non plus. C'est un simple outil de zonage, qui n'est pas visible sur le terrain (pas de panneaux...).

« C'est un outil législatif, très politisé [...], on le considère vraiment comme un outil d'urbanisme et pas comme de la communication. » (CCET.)

Certains agriculteurs déplorent le fait qu'elle ne soit pas assez mise en valeur, notamment auprès de la clientèle.

«Elle n'est même pas mentionnée sur le site de la cave coopérative.» (Mme L., viticultrice à Montlouis-sur-Loire.)

Certains agriculteurs n'ayant pas adhéré au syndicat des vins, ou d'autres qui ne se sentent pas directement touchés par l'étalement urbain, ne savent même pas si leurs parcelles sont classées en ZAP ou pas. La ZAP aurait donc d'avantage d'impact sur l'urbanisme que sur l'économie viticole, d'après certains vignerons.

À travers la délimitation de la ZAP, la commune a pu définir ses besoins en matière d'urbanisation pour les 20 ans à venir, tout en protégeant durablement les parties les plus importantes du vignoble contre l'étalement urbain. Elle a aussi, grâce à cette procédure, défini les finalités de cette protection et reconnu la fonction économique du vignoble tout en mettant en avant la protection du cadre de vie, du patrimoine et du paysage.

Cette ZAP fait partie d'un projet d'une plus grande envergure, le projet agro-urbain qui vise à permettre un développement équilibré des cinq communes de la CCET. Aussi, elle s'est dotée d'un programme visant le développement durable et la protection des espaces agricoles et des paysages ruraux. Celui-ci a été élaboré suite à un diagnostic mené par la direction départementale de l'Agriculture et de la Forêt (DDAF), le Centre d'études supérieures d'aménagement, et l'École nationale supérieure de la nature et du 
paysage, sous la direction d'un comité de pilotage composé d'élus des différentes communes (auquel s'est associé un élu de Lussault-sur-Loire), ainsi que des représentants de la chambre d'agriculture, de la Société d'améngement foncier et d'établissement rural (Safer), de l'Inao, du syndicat des vins de Montlouis-sur-Loire, du syndicat des maraîchers, de la maison de la Loire et de l'agence d'urbanisme.

Il a pour objectif d'œuvrer en faveur du maintien et du développement de l'agriculture en luttant contre les friches, en organisant la gestion de l'espace dans le double respect de la production agricole et de la préservation du cadre de vie, en affirmant la vocation agricole à long terme de certains terroirs (c'est ici que s'inscrit la ZAP), en favorisant et en vulgarisant l'agriculture raisonnée, la diversification agricole et en créant une structure de dialogue. Ce projet concerne toute l'activité agricole sur le territoire de la CCET. Pour ce qui concerne la viticulture, ce plan prévoit des remembrements des parcelles viticoles, un accompagnement dans l'installation des jeunes viticulteurs, un projet de zone d'activité viticole. Le projet souhaite aussi sensibiliser la population pour permettre aux habitants de découvrir les différents paysages et les activités agricoles qui les façonnent.

\section{L'AOC Vouvray : Une action en ordre dispersé}

À la différence de Montlouis, jusqu'au début 2013, il n'existait pas d'outils spécifiques de protection de l'AOC. Les règles d'urbanisme, fixées au niveau communal, étaient les seules appliquées. Toutes les municipalités qui se sont succédé ont toujours eu pour volonté de protéger les zones vigneronnes. Il y a en effet un lien étroit entre les élus locaux et la viticulture. Ainsi les conseils municipaux sont en très forte majorité composés d'élus viticulteurs ou vignerons, à l'image de Vouvray où le maire, également président de la CCV, est aussi viticulteur.

«On a toujours lutté contre les constructions. Lors de la révision du POS qui est devenu le PLU en 2007 nous avons même remis en zone viticole des parcelles qui avaient été classées en zone U pour suivre au maximum les limites de l'appellation. [...] C'est surtout une volonté politique, une volonté de protéger le vignoble compte tenu de tout ce que ça représente au niveau économique et au niveau touristique aussi. » (Maire de Vouvray.)

Les conseils municipaux mettent en avant la richesse économique que constitue le vignoble en termes de nombre d'exploitations et de nombre d'emplois. Si les parcelles AOC sont préservées de toute urbanisation, les espaces céréaliers du plateau sont clairement utilisés comme réserve foncière. La collectivité avance que les vignobles en appellation, à la différence des grandes cultures, sont liés à des terroirs, qu'ils ne sont pas délocalisables et sont aussi vecteurs d'une identité forte, d'où la grande volonté de les épargner.

Malgré tout, l'extension urbaine constitue « le plus gros danger pour notre vignoble car il se trouve très proche de Tours. Certaines communes gèrent bien cette pression, d'autres semblent plus fragiles, cela dépend des maires et des conseils municipaux " (monsieur V. viticulteur de Vernou-sur-Brenne). Pour lui, la menace liée à l'étalement est bien présente "surtout que certains vignerons sont les premiers à penser pouvoir vendre des terrains ". Mais le syndicat des vins veille et suit de près les révisions des documents d'urbanisme pour éviter que les parcelles enclavées ne deviennent constructibles. 
Depuis le 13 février 2013 les communes de Rochecorbon et Parçay-Meslay se sont dotées à leur tour d'une ZAP. Les objectifs affichés sont la maîtrise de l'urbanisation, la dynamisation de l'agriculture (conserver les terres agricoles et permettre la circulation du matériel), la valorisation des paysages pour maintenir un cadre de vie et la conservation du patrimoine bâti et non bâti. La volonté est d'afficher une lisibilité à long terme de la protection agricole, de proposer un projet équilibré, de partir d'un périmètre le plus étendu possible et de l'adapter aux enjeux de développement des communes à long terme. Elle couvre donc bien plus que le vignoble puisqu'elle s'étend sur des terres agricoles et naturelles.

«La ZAP c'est agricole, ce n'est pas que l'AOC. À Rochecorbon c'est 350 hectares de vignes, le reste ce sont des champs, si l'on enlève le bourg. » (Monsieur L. vigneron à Rochecorbon.)

Mais contrairement à la ZAP de Montlouis, celle de Rochecorbon et de Parçay-Meslay ne bénéficie pas d'une adhésion de tous les acteurs du territoire. Elle n'est soutenue ni par la CCV ni par le syndicat des vignerons et fait l'objet d'une vive polémique. Des raisons politiques sont évoquées. Les deux communes souhaitent depuis plusieurs années rejoindre la communauté d'agglomération Tour(s)Plus. Les habitants de ces communes sont réticents car se posent pour eux le devenir des terres agricoles et donc de leur qualité de vie. Intégrer Tour(s)Plus pourrait accentuer l'artificialisation des terres communales. Alors, pour les municipalités, protéger ces terres agricoles est le préalable à l'intégration des deux communes à la communauté d'agglomération Tour(s)Plus.

« Il y une raison politique à la ZAP. C'est une façon de rassurer les gens par rapport

à Tour(s)Plus et à l'agriculture, de leur dire qu'avec une ZAP ils sont tranquilles pendant 20 ans. » (CCV.)

Les communes de Rochecorbon et de Parcay-Meslay ont rejoint Tour(s)Plus le $1^{\text {er }}$ janvier 2014. Derrière cette ZAP, se cache un double enjeu.

«La communauté de communes telle qu'elle est actuellement a une certaine identité, une cohérence, une continuité territoriale. Et il y a cette appellation qui fait le lien entre les communes, et les personnes qui sont attachées à ce terroir trouvent dommage que ces communes aillent dans Tour(s)Plus, sachant que les compétences sont différentes, les objectifs seront donc aussi différents. » (Mairie de Vouvray.)

Certains opposants au rapprochement avec Tour(s)Plus rappellent la situation de la commune de Sainte-Radegonde, rattachée à Tours dans les années 1970, et qui aujourd'hui ne possède plus qu'un hectare et demi de vigne, le reste s'étant urbanisé. Les vignerons craignent donc que le même schéma se reproduise avec Rochecorbon et Parçay-Meslay. Si les deux communes de la CCV les plus proches de Tours souhaitent intégrer la communauté d'agglomération Tour(s)Plus, les autres sont plus attachées à leur identité rurale et craignent de se voir dissoutes dans un ensemble urbain trop vaste.

Le sujet de la ZAP est très clivant auprès de l'ensemble des acteurs interrogés. Le syndicat est contre la ZAP car il ne veut pas voir transformer les parcelles AOC en " réserve d'Indiens » pour reprendre les mots de son président. Celui-ci souhaite que l'AOC soit maintenue, parce qu'elle représente «une force économique, un atout touristique et non pour des raisons politiques...». Pour les municipalités de Rochecorbon et de Parçay-Meslay, la non-adhésion de certains peut s'expliquer pour 
des raisons de spéculation foncière. La souplesse du PLU par rapport à la ZAP permet de rendre constructibles certains terrains agricoles. Les viticulteurs admettent garder cette possibilité pour renforcer l'activité en cas de force majeure.

«On a eu la chance depuis des décennies d'avoir des municipalités qui ont eu pour objectif de protéger le vignoble de Vouvray. Hormis quelques parcelles au nord de la limite d'appellation de Vouvray, où il y a eu des entreprises vouvrillones qui étaient en centre-bourg et qui avaient besoin de se développer, les parcelles AOC ont été préservées. " (Mairie de Vouvray.)

La question du périphérique de Tours. Il s'agit d'un projet d'aménagement routier dont les premiers tronçons ont vu le jour à la fin des années 1960, mais qui ne devrait être totalement achevé que vers 2030. Les parties sud et sud-ouest du périphérique sont achevées depuis la fin des années 1990. La partie nord-ouest, quant à elle, a été achevée et ouverte à la circulation en 2011. Mais le bouclage nord-est est plus complexe car il doit passer par le vignoble de Vouvray.

Figure 2. L'enjeu du périphérique

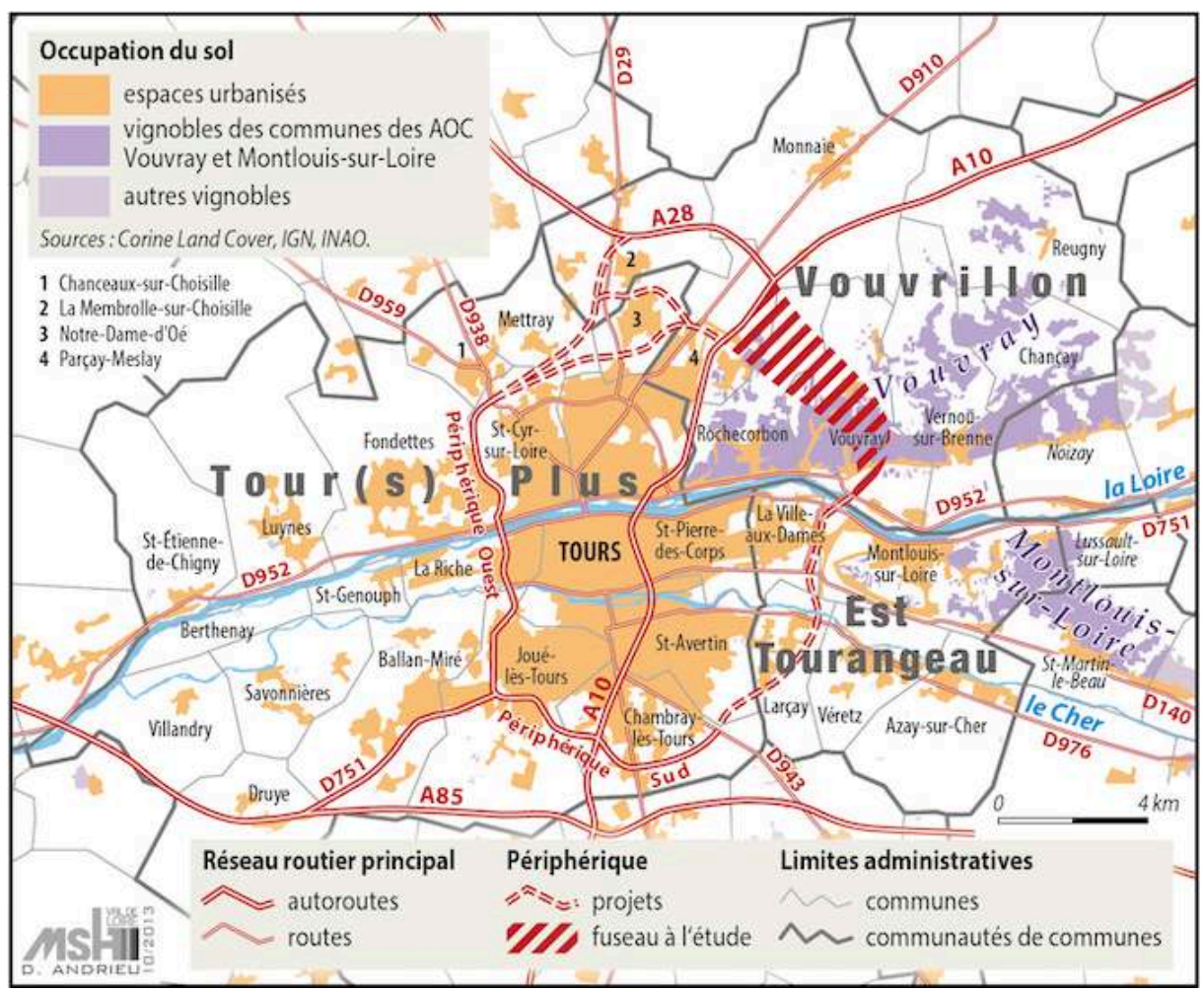

Le secteur viticole, bien organisé et très influent, a déjà obtenu la mise en tunnel de la ligne à grande vitesse (LGV) Atlantique en 1989. Depuis, la vallée de la Loire a été inscrite sur la liste du patrimoine mondial de l'Unesco, favorisant de fortes contraintes de préservation du paysage comme le propose le plan gestion, même si l'inscription n'engendre pas de dispositifs réglementaires supplémentaires. Cette zone fait également partie des variantes envisagées pour l'A10 bis (qui aujourd'hui passe dans Tours posant de sérieux problèmes de pollutions), et les deux infrastructures pourraient être fusionnées pour franchir ce point sensible. Devant l'ampleur des difficultés, la réalisation de ce tronçon n'est pour l'heure ni planifiée ni certaine de voir le jour, mais le syndicat et la CCV veillent. Ils sont mobilisés contre le projet qui 
provoquerait des suppressions de parcelles viticoles à Rochecorbon et Parçay-Meslay, environ 450 ha, soit près du quart de l'AOC (voir figure ci-dessus).

« Pour l'instant on ne sait pas où il va passer exactement. Le périphérique ne fait pas vraiment nos affaires, avec des sorties tous les 500 mètres, avec un rond-point, et toutes sortes de constructions, hôtels et autres, et ça fait des voitures partout. » (Monsieur L., viticulteur à Rochecorbon.)

Le rattachement des deux communes à Tour(s)Plus rendrait alors moins efficiente l'opposition de la CCV et du syndicat au projet.

\section{Malgré tout, une mise en valeur du paysage} tensions dans le vignoble de Vouvray, notre travail fait ressortir une constante, un point d'accord partagé par tous: la place centrale du paysage dans la stratégie de résistance et de pérennisation de ces vignobles périurbains. Trois points ressortent clairement.

\section{L'appellation d'origine contrôlée, un moyen de protection majeur}

Il est évident que des terroirs viticoles reconnus comme de grande qualité par les appellations bénéficieront d'une valeur foncière supérieure qui favorisera le maintien d'une activité agricole et rendra moins attractive l'hypothèse de leur constructibilité (Vincent, 2009; Pères, 2007, Morlat, 2001 ; Maby, 2003). La survie de la viticulture de qualité est fondamentalement dépendante $\mathrm{du}$ maintien des équilibres environnementaux puisque, en aire AOC, l'environnement est à la source de la qualité du vin par la valeur agronomique et la typicité œnologique des terroirs. Au-delà de la seule valeur matérielle du vin, constituée par toute une série de facteurs techniques vitivinicoles (sols, cépages, modes de conduite et de vinification...), se développe une valeur immatérielle symbolique et culturelle qui constitue l'atout concurrentiel éminent des AOC (Maby, 2008).

Mais pour les AOC comme Vouvray et Montlouis-sur-Loire qui ne sont pas assez réputées pour se suffire en termes de protection, la résistance viticole, liée à l'appartenance à l'AOC, est un combat quotidien. Il faut faire vivre et faire connaître l'AOC. Les vignerons en sont conscients :

« L'appartenance à l'appellation d'origine contrôlée, c'est ce qui permet de certifier la qualité du vin. C'est grâce à elle qu'on reconnaît la qualité du vin produit et donc que les vignobles peuvent se développer économiquement. Plus on parle de l'appellation plus elle est connue au niveau national mais aussi international, ce qui permet même d'exporter les vins et d'élargir sa clientèle. Plus l'AOC est connue et se porte bien, plus elle résistera à la pression immobilière.» (Monsieur C., viticulteur sur Vernou-sur-Brenne.)

Pour madame D., vigneronne à Vouvray :

«L'appellation est le principal atout pour la mise en valeur du vin.»

C'est le rôle des syndicats des vins de promouvoir et de faire connaître l'appellation, à travers des projets comme des portes ouvertes, des dégustations. Ils sont très fortement appuyés par les collectivités et la mission Val de Loire, le syndicat mixte interrégional qui a en charge de faire vivre l'inscription au patrimoine mondial de l'Unesco. 
Monsieur M., vigneron à Montlouis, décrit les actions effectuées par le syndicat pour promouvoir l'appellation :

«Montlouis, c'était [...] une petite appellation de rien du tout dans le centre de la France, mais on a été à l'initiative de pleins de projets, comme il y a 25 ans, les matinées découvertes. À l'époque j'étais secrétaire du syndicat, on faisait venir des prescripteurs de toute la France, on faisait des dégustations des meilleurs crus de l'appellation et après on faisait un repas dans le cadre du château de la Bourdaisière, avec des grandes toques de Touraine. Il faut savoir que c'est une idée qui fonctionne très bien et qui a été reprise en Champagne. Nous, ça fait déjà pratiquement 10 ans qu'on le faisait, et on l'a fait pendant une quinzaine d'années, mais ça coûte très très cher. Chaque plat et chaque bouteille étaient commentés par un œnologue et un grand sommelier. [...] C'est vrai que ça, ça donne une bonne image de l'appellation Montlouis vis-à-vis de la restauration générale et des prescripteurs. » (Monsieur M., vigneron à Montlouis.)

Et Monsieur D., viticulteur à Vouvray :

«L'appellation, j'y crois. Il faut se battre pour la faire connaître, au sein du syndicat mais aussi individuellement pour essayer d'améliorer le côté qualitatif aussi, car on est confronté maintenant au marché mondial.» (Monsieur D., viticulteur à Vouvray.)

Les deux AOC s'accordent à dire que c'est en améliorant la qualité du paysage viticole que l'appellation aura plus de reconnaissance et donc que le vignoble pourra peser contre la menace liée à l'étalement urbain. Pour certains, c'est même l'élément décisif pour la protection du vignoble contre l'imperméabilisation des sols.

«Si vous pouviez avoir une vue aérienne de Tours vous verriez que si Rochecorbon n'avait pas eu l'AOC on ne verrait plus beaucoup de champs aux alentours de la ville. Mais comme on pèse économiquement ils sont obligés de nous écouter!» (Monsieur L., viticulteur à Rochecorbon.)

Ainsi la notion d'AOC offre l'avantage éminent de pouvoir garantir une origine et donc, de fait, d'associer un type paysager particulier à chaque vin. Cette esthétique paysagère est déjà un critère de qualité, sous-entendu dans l'éventail des valeurs d'une AOC. Ce sous-entendu mérite d'être mis en valeur.

\section{La conservation et la mise en valeur du paysage viticole}

La vigne est un marqueur identitaire fort du Val de Loire (Yengué, 2013). Le vignoble se décline en une succession d'AOC, de vins et de paysages viticoles différents. Ces territoires viticoles ont vécu d'importantes évolutions paysagères, notamment avec la mécanisation de l'agriculture (Dion, 1990). Jacques Maby (2001) a étudié les paysages viticoles et les enjeux qui s'en dégagent. Selon lui, le monde viticole est de plus en plus concerné par la question paysagère, il s'agit même de la survie de l'image positive dont bénéficient les appellations d'origine contrôlée. Les paysages sont composés d'éléments qui renvoient à des références socioculturelles fortes, susceptibles de modeler l'image d'un produit et d'en déterminer la notoriété et le prix. Le paysage est donc un outil de qualification des produits du terroir ainsi qu'un instrument de communication et de promotion pour ceux-ci.

Le paysage viticole est fondé sur des éléments naturels (le cep de vigne, le sol, la topographie...). Cependant, tout dans le paysage du vignoble dénote l'action humaine : les formes du parcellaire, les alignements de haies, le réseau de la voirie, les éléments du bâti inséré, les particularités des modes de conduite culturale... C'est donc une 
synthèse de l'action naturelle et humaine qui crée ce paysage si particulier. Ce dernier doit donc garder son équilibre entre les éléments naturels et humains. Ainsi la protection des paysages viticoles n'est ni une fin en soi à vocation promotionnelle ni une passion esthétique désintéressée, mais procède de la logique de préservation de cet équilibre que rompt l'étalement urbain.

La richesse paysagère fait non seulement la preuve de la qualité du territoire mais aussi celle du vin. Cette richesse s'intègre au prix du vin et constitue une grande partie de la valeur ajoutée de la production viticole. La mise en valeur du paysage, et donc du patrimoine viticole, est primordiale car elle permet d'affirmer l'identité collective des vignerons.

Afin de protéger et de gérer ces paysages, une charte paysagère, la charte de Fontevraud, a été créée (Asselin et al., 2003, Rochard et al., 2008). Cette charte propose la création, à partir du Val de Loire, d'un réseau international porté par les acteurs des territoires de la vigne et du vin, avec pour objectif un engagement d'excellence, de protection, de gestion et d'aménagement des paysages de ces territoires dans le respect de leurs valeurs naturelles et culturelles. Cette charte s'attache à valoriser la qualité environnementale, culturelle, touristique et économique de ces paysages. Elle a été élaborée par InterLoire (l'interprofession des vins du Val de Loire), la mission Val de Loire, et signée en 2003 par les ministères de l'Agriculture et de l'Environnement, l'office international du Vin, Inao, la Confédération des vignerons du Val de Loire et InterLoire. Elle s'appuie sur un partenariat entre les syndicats viticoles, les collectivités locales, les professionnels du tourisme et les monuments patrimoniaux. L'enjeu est d'engager une démarche conjuguant l'optimisation de la production vinicole et la gestion des paysages de ces territoires. Il s'agit d'associer une qualité de démarche environnementale à une valorisation culturelle et touristique de ces paysages, dans le cadre d'un réseau international d'excellence. La charte stipule qu'il est nécessaire d'éviter une banalisation des paysages, une fermeture du paysage due à l'abandon de certaines terres et un développement mal maîtrisé de l'urbanisation.

Dans les AOC de Montlouis-sur-Loire et de Vouvray, la richesse patrimoniale, garante de la qualité paysagère, constitue une grande partie de la valeur ajoutée de la production viticole des territoires. Des démarches volontaires (collectivités, syndicats, vignerons) se renforcent pour une gestion du paysage devant assurer l'insertion harmonieuse et le respect des éléments du patrimoine viticole :

- nombreux sont les ronds-points paysagers sur le thème du vin : loges de vigne, rangs de vigne, pressoirs, grappes de raisins géantes, etc., embellissent les terre-pleins centraux ;

- les loges de vignes sont réhabilitées avec des financements publics et privés ;

- la CCET, en partenariat avec le conseil général d'Indre-et-Loire et la Fédération des chasseurs, a mis en place un financement pour la réalisation de jachères fleuries. L'objectif est de lutter contre la banalisation des paysages et de favoriser la biodiversité tout en replaçant les agriculteurs et viticulteurs comme acteurs de l'aménagement et de l'entretien des espaces agricoles;

- les habitations troglodytes sont rachetées par des coopératives ou des offices de tourisme pour devenir des vitrines de l'activité viticole et vigneronne. C'est notamment le cas de la cave des producteurs de Montlouis ou de l'office du tourisme de Montlouis sur Loire.

Ce paysage viticole, avec son petit patrimoine réhabilité avec charme, avec ses rangs de vigne qui soulignent l'ondulation du relief, devient un argument politique pour vanter la qualité de vie dans ces « communes urbaines à la campagne». La zone de protection 
du patrimoine architectural, urbain et paysager de Rochecorbon, entrée en vigueur le 22 janvier 2008, participe de cette patrimonialisation du paysage en insistant sur la préservation du plateau viticole.

\section{L'œnotourisme, facteur de développement local}

Dans le Val de Loire inscrit au patrimoine de l'Unesco, le vignoble est d'emblée positionné comme un atout touristique pour le territoire (Amalric et al., 2011; Verdelli et al., 2010). Sur les deux AOC, les produits du vignoble (vin et paysages) sont depuis une dizaine d'années le support d'un tourisme organisé conjointement par les collectivités, les syndicats et les vignerons, et mis en scène dans les offices du tourisme. À Montlouissur-Loire, l'office du tourisme se situe dans un emplacement privilégié en terme de patrimoine paysager, puisqu'il s'est logé dans le tuffeau (cave troglodyte) en bord de Loire. Cette implantation stratégique assure une bonne visibilité de la dimension paysagère de l'appellation et constitue un élément favorable à la promotion de celle-ci.

À Montlouis, l'œnotourisme est favorisé entre autres par le passage de l'itinéraire La Loire à vélo dans les vignes de la commune. Cette piste cyclable était censée longer la Loire de Nevers à Nantes. Lors de la construction de son tracé à l'est de Tours (2008), les élus montlouisiens et le syndicat ont œuvré pour qu'il devienne l'une des vitrines du vignoble en le détournant du fleuve. Si les viticulteurs étaient réticents au passage de la piste cyclable dans leurs vignes, ils commencent à reconnaître les retombées positives pour leurs exploitations.

La Loire à vélo ne passant pas dans l'AOC Vouvray, la CCV, avec le partenariat de la communauté de communes du Val d'Amboise, a mis en place un itinéraire cyclable, comme l'explique le président de la CCV et maire de Vouvray :

«La Loire à vélo ne passe pas à Vouvray, à une époque il en était question. Se posait un problème entre Vouvray et Vernou-sur-Brenne, c'était très difficile à faire et très coûteux, ce qui a dû faire changer d'avis les financeurs. Nous, ce qu'on a fait l'an dernier, c'est qu'on a mis en place un itinéraire cyclable de l'ouest de la commune de Rochecorbon, on l'a fait en partenariat avec la communauté de commune du Val d'Amboise, et donc ça va rejoindre la gare d'Amboise, à travers les différentes vallées, vallée de la Loire, vallée de la Brenne... et le vignoble. »

L'AOC Vouvray a une configuration topographique qui ne rend pas visible les vignes. Elles sont positionnées sur les interfluves alors que les voies d'accès sont dans les vallées. Tout un système de panneaux indicateurs a donc été déployé sur les quais de Loire indiquant les chemins de randonnées et les caves des viticulteurs.

«On organise des circuits de découverte, parce que la problématique sur l'AOC de Vouvray, c'est que l'on est sur les grandes voies qui desservent le territoire sans rencontrer une parcelle de vigne. La particularité, c'est qu'il y a plein de petites vallées et les vignes sont sur le coteau au-dessus, donc quand on passe en bas dans la vallée on ne voit pas les vignes, donc faut leur faire découvrir.» (Maire de Vouvray et président de la CCV.)

47 Tous les acteurs interrogés admettent l'importance du tourisme et du paysage pour l'activité mais aussi pour le territoire. Le tourisme constitue donc une part importante de l'activité vigneronne.

"On se déplace, on fait des petits salons, on organise des portes ouvertes chaque année et ensuite toute l'année on reçoit des clients, des touristes à vélo, on fait visiter le chai, les caves troglodytes et on fait des dégustations. " (Monsieur S. viticulteur à Montlouis-sur-Loire.) 
«Le tourisme a été le moyen premier en Touraine, avec les châteaux de la Loire, de promotion de nos produits à nous. C'est toujours le cas, et ça le restera tant que les châteaux et nos beaux paysages seront là pour attirer les touristes.» (Mme D. viticultrice à Vouvray.) l'extension urbaine. Malgré tout, ces vignobles résistent et sont aujourd'hui les surfaces
viticoles les plus importantes de l'agglomération tourangelle. Les recettes de cette forte résistance diffèrent entre les deux terroirs. Montlouis-sur-Loire fait le choix de mobiliser les outils réglementaires comme la ZAP. Vouvray souhaite garder de la souplesse et faire confiance à la corporation. Dans les deux cas, le paysage reste au cœur de toutes les stratégies. Il représente une valeur identitaire forte et un poids économique croissant dans un site inscrit au patrimoine mondial de l'Unesco et qui veut faire du tourisme un levier de développement. La préservation du vignoble renforce la qualité de vie dans ces communes, elle-même facteur de pression foncière qui demande à être régulée par une protection plus stricte. Un système se construit donc autour du paysage, englobant les thématiques des trames vertes et bleues, d'agriculture urbaine et de nature en ville.

\section{BIBLIOGRAPHIE}

Amalric, M., Servain-Courant, A., Verdelli, L., Yengué, J.-L., « Stratégies de protection patrimoniale et valorisation touristique des paysages culturels, trois démarches significatives. Tourisme et territoire ", dans Bleton-Ruget, A., Commerçon, N., et Lefort, I., (dir), Tourismes et Territoires, Mâcon, Institut de recherche du Val de Saône-Mâconnais, 2011.

Ambroise, R., «Les pressions et les enjeux paysagers concernant les sites viticoles », Étude thématique, Les paysages culturels viticoles dans le cadre de la convention du Ppatrimoine mondial de l'Unesco, Paris, Icomos, 2005, p. 51-55

Asselin, C., Laidet, M., « Présentation de la charte de Fontevraud », Actes du colloque international « Paysages de vignes et de vins - Patrimoine enjeux valorisation », Fontevraud, Accentis, 2003, 313 p.

Babonaux, Y., « L'évolution actuelle du vignoble tourangeau », Norois, n 53, 1967, p. 6-68.

Barthelemy, D., Dagot, H., Wavreski, P., « Valeur et rentabilité des vignes et des exploitations viticoles en Bourgogne ", Économie et Sociologie rurales, Dijon, Inra, 2000, 129 p.

Chaballier, C., « Pressions urbaines et résistances viticoles. Quelques enseignements dans les AOC de Vouvray et de Montlouis-sur-Loire ", mémoire de M1 géographie, université de Tours, 2010.

Cavailhes, J., « L'extension des villes et la périurbanisation », Villes et Économie, Paris, La Documentation française, 2004, p. 157-184.

Dion, R., Le Paysage et la Vigne. Essais de géographie historique, Paris, Payot, 1990. 
Dion, R., Le Val de Loire. Étude de géographie régionale, Tours, Arrault, 1934.

Laroche, B.,Thorette, J.,Lacassin, J-CL., « L'artificialisation des sols : pressions urbaines et inventaire des sols ", Étude et gestion des sols, volume XIII-3, 2006 p. 223-235.

Maby, J., « Vin et identité spatiale, l'exemple du Maipo Alto. Chili », Revue historiens et géographes, $\mathrm{n}^{\circ} 404,2008$, p. 91-100.

Maby J., « Les enjeux paysagers viticoles », Actes du symposium international « Terroirs et zonage vitivinicole », Avignon, Office international de la vigne et du vin, 2003, p. 823-831.

Maby, J., « Paysages et imaginaire : l'exploitation de nouvelles valeurs ajoutées dans les terroirs viticoles », Annales de géographie, $n^{\circ}$ 624, 2001, p. 198-211.

Morlat, R. (dir.), Terroirs viticoles : étude et valorisation, Paris, Éditions Oenoplurimédia, coll. « Avenir œnologie », 2001.

Pères, S., « La résistance des espaces viticoles à l'extension urbaine : le cas du vignoble de Bordeaux », Revue d'économie régionale et urbaine, $\mathrm{n}^{\circ}$ 1, Paris, Armand-Colin, 2009, p. 155-177.

Pères, S., «La Vigne et la ville : forme urbaine et usage des sols », Bordeaux 4, thèse ès sciences économiques, 2007.

Rochard, J., Lasnier, A., Herbin, C., Ambroise, R. « La charte international de Fontevraud en faveur des paysages viticoles ", VII congrès international des terroirs viticoles, Nyon, 2008, p. 710-713.

Serreno, J., Vianey, V. « Les zones agricoles protégées : figer de l'espace agricole ou organiser le territoire pour un projet urbain ?", Géographie, Économie, Société, 2007/4, vol. 9, 2007 p. 419-438.

Verdelli, L., Yengué, J.-L.,Servain, S., « Ressources patrimoniales et dispositifs touristiques innovants. Le cas de la France et du Portugal à travers deux communes rurales appartenant à des paysages culturels Unesco ", dans Berger, A., Chevalier, P., Cortes, G., Dedeire, M. (dir.), Patrimoine, Héritages et Développement rural en Europe, Paris, L'Harmattan, 2010.

Vincent, E., « Le rôle des vignerons dans la conservation des paysages ", Paysages et Patrimoines viticoles, Dijon, chaire Unesco Culture et traditions du Vin, 2009, 16 p.

Yengué, J.-L., « Vinyard and Landscape in the Loire Valley (France) », Journal of Agricultural Science and Technology, 2013, p. 226-230.

Yengué, J-L., Servain, S., Boutin, D. « Viticulture et évolution des paysages en Loire moyenne, Vernou-sur-brenne (Indre-et-Loire, France) », Actes du colloque international « Paysages de Vignes et de Vins - Patrimoine enjeux valorisation », Fontevraud, Accentis, 2003, p. 175-177.

\section{NOTES}

1. http://www.vins-vouvray.com/index.php; https://www.inao.gouv.fr

2. https://www.vinsvaldeloire.fr/

3. Il s'agit ici d'une démarche exploratoire concernant la perception des viticulteurs sur la protection de leur terroir 


\section{RÉSUMÉS}

Cette étude montre deux vignobles périurbains (les appellations d'origine contrôlée Montlouis et Vouvray) touchés de plein fouet par l'extension urbaine de l'agglomération tourangelle (Indreet-Loire). Elle n'a cessé de s'accroître depuis la fin de la Seconde Guerre mondiale avec une accélération à partir des années 1970 et, depuis 30 ans, une perte de 8000 ha de surface agricole utile et de $58 \%$ des exploitations agricoles. Malgré tout, ces vignobles résistent et sont aujourd'hui les surfaces viticoles les plus importantes de l'agglomération tourangelle. Des entretiens auprès des acteurs locaux ont permis de décrypter les stratégies de résistance misent en place ici face à la pression urbaine. Si les approches, les outils mobilisés et l'implication des acteurs sont différents dans les deux cas, il ressort très nettement qu'ici, le paysage et sa mise en patrimoine deviennent le principal rempart à l'urbanisation.

This study presents two peri-urban vineyards (the controlled designations of origin of Montlouis and Vouvray) directly impacted by the urban sprawl of the agglomeration of Tours (Indre-etLoire). The agglomeration has grown continuously since the end of the Second World War with a period of marked acceleration in the 1970s and a loss of 8000 ha of agricultural land and of $58 \%$ of farms in the last 30 years. In spite of this, these vineyards have resisted and are a part of the largest wine growing areas in the agglomeration of Tours. Interviews with local stakeholders made it possible to understand the strategies for resisting urban pressure. Although the approaches and tools employed as well as the implication of these stakeholders were different in each of the cases, clearly the landscape and recognising it as a heritage proved to be the main defence against urbanisation.

\section{INDEX}

Keywords : controlled designation of origin (in French AOC), landscape, heritage, promotion of the vineyard, urban pressure

Mots-clés : AOC, paysage, patrimoine, valorisation du vignoble, pression urbaine

\section{AUTEURS}

\section{JEAN-LOUIS YENGUÉ}

Jean-Louis Yengué est géographe, maître de conférences, UMR Citeres CNRS/université de Tours. yengue[at]univ-tours[dot]fr

\section{CLOTHILDE CHABALLIER}

Clotilde Chaballier est titulaire d'un master 2 environnement, territoire et paysage, université de Tours et apprentie au CAUE 41.

clotilde.chaballier[at]hotmail[dot]fr 